\title{
Effectiveness of HP-hMG vs r-FSH in Patients undergoing IVF/ICSI Cycles with Moderate Male Factor Infertility
}

\author{
Rutvij Jay Dalal, Hrishikesh Pai, Nandita Palshetkar
}

\begin{abstract}
Aim: The aim of this case-control study was to compare the efficacy of highly purified human menopausal gonadotropin (HP$\mathrm{hMG}$ ) vs recombinant follicle stimulating hormone ( $r-\mathrm{FSH})$ treatments following $\mathrm{GnRH}$ agonist suppression in patients undergoing intracytoplasmic sperm injection (ICSI) with moderate male factor infertility in terms of oocyte and embryo quality and clinical pregnancy outcomes.
\end{abstract}

Materials and methods: A total of 240 infertile women were treated with HP-hMG group (n: 120 patients) or ( $\mathrm{r}-\mathrm{FSH}$ group, $\mathrm{n}$ : 120 patients) following $\mathrm{GnRH}$ agonist suppression (long regimen). Inclusion criteria for the study groups were infertility due to moderate oligoastheno-teratospermia with no associated female infertility factor, previous ART cycles $<2$, female patients aged 19 to 35 years with normal basal FSH, regular ovulatory cycles and $\mathrm{BMI}<30 \mathrm{~kg} / \mathrm{m}^{2}$.

Results: Treatment durations and gonadotropin doses were similar in both groups. Cycle cancellation rates, clinical pregnancy and miscarriage rates, total and metaphase II oocytes retrieved, fertilization rate, number of embryos transferred were all similar in both groups. The clinical pregnancy rates were $45.9 \%$ (n: 50/109) in the r-FSH group and 40.4\% (n: 44/109) in the HP-hMG group.

Conclusion: HP-hMG is effective as r-FSH in terms of oocyte and embryo quality and clinical pregnancy outcomes in patients undergoing ICSI with moderate male factor infertility.

Keywords: Male factor infertility, Intracytoplasmic sperm injection, Highly purified hMG, Recombinant FSH, GnRH agonist.

How to cite this article: Dalal RJ, Pai H, Palshetkar N. Effectiveness of HP-hMG vs r-FSH in Patients undergoing IVF/ICSI Cycles with Moderate Male Factor Infertility. Int J Infertility Fetal Med 2012;3(2):51-56.

Source of support: Nil

Conflict of interest: None

\section{INTRODUCTION}

In recent years, induction of ovulation has shown major advances, with multiple products commercially available and the focus of ovarian stimulation has shifted from trying to obtain the maximum possible number of oocytes to trying to obtain an adequate cohort of good-quality embryos, i.e. from quantity to quality. ${ }^{1}$ Urinary products include human

Date of Received: 18-07-12

Date of Acceptance: 14-08-12

Date of Publication: May 2012 menopausal gonadotropins (hMG), urinary follicle stimulating hormone $(\mathrm{FSH})(\mathrm{uFSH})$ and human chorionic gonadotropin (hCG). More recently, recombinant preparations such as r-FSH and rLH have entered the market. Finally, highly purified (HP) hMG, in which the purification process allows its administration via the subcutaneous route, is the latest addition to this family of infertility drugs. HP-hMG and r-FSH have been widely and successfully used for ovarian stimulation in infertile women undergoing treatment for in vitro fertilization/intracytoplasmic sperm injection (IVF/ICSI) and embryo transfer.

It is well known that the quantitative aspects can be modulated by the doses of gonadotropins, the type of gonadotropin used and by the endocrine environment associated with stimulation. ${ }^{2-5}$ Randomized controlled trials comparing gonadotropin preparations have primarily focused on clinical aspects and have been designed to evaluate the number of oocytes retrieved or to a lesser extent pregnancy rates.

Several studies comparing the outcome of r-FSH and hMG have been reported, most of which were performed in women undergoing pituitary down-regulation with a $\mathrm{GnRH}$ agonist long protocol. ${ }^{6-13}$ Recent meta-analyses have demonstrated that hMG was not inferior to r-FSH with regard to pregnancy and live birth rates. ${ }^{14,15}$ Cochrane review found a borderline significant difference of a $5 \%$ higher clinical pregnancy rate in women stimulated with menotropins (27\%) compared with r-FSH $(22 \%) .{ }^{16}$ The authors further noted that additional large randomized trials were needed to precisely estimate any difference between menotropins and r-FSH. Recently, it was confirmed that a better outcome in terms of the live birth rate is obtained when Hp-hMG was used for ovarian stimulation compared with r-FSH in the GnRH agonist long protocol. ${ }^{17}$

The aim of the present case control study was to compare the efficacy of HP-hMG, which combines FSH and HCGdriven $\mathrm{LH}$ activities vs r-FSH alone in patients undergoing ICSI with moderate male factor infertility with a view to oocyte and embryo quality and IVF treatment outcome.

\section{MATERIALS AND METHODS}

A total of 240 infertile women were treated with HP-hMG (HP-hMG group, n: 120 patients) or r-FSH (r-FSH group, 
$\mathrm{n}: 120$ patients) following GnRH agonist suppression (long regimen).

Inclusion criteria for the study groups were infertility due to moderate oligoasthenoteratospermia with no associated female infertility factor, previous ART cycles $<2$. Oligoasthenoteratospermic patient was defined as sperm concentration between $5 \times 10^{6}$ and $15 \times 10^{6} / \mathrm{ml}^{18}$

Patients were selected if they met all the following inclusion criteria: Women with good physical and mental health, aged 19 to 35 years; regular menstrual cycles ranging from 21 to 35 days; BMI $<30 \mathrm{~kg} / \mathrm{m}^{2}$; normal basal serum FSH (1-12 IU/1) and estradiol (E2, $\leq 75 \mathrm{pg} / \mathrm{ml})$ levels determined on day 3 of the cycle previous to controlled ovarian stimulation (COS), uterus consistent with expected normal function, presence of both ovaries without evidence of abnormality and no adnexal pathology assessed by transvaginal ultrasound.

The exclusion criteria were: Patients with a history of recurrent pregnancy loss; any significant systemic disease, endocrine or metabolic disorder; having concomitant medication interfering with the purposes of the study; patients who have received any ovulation induction drug within 1 month before their inclusion in the study. Patients with polycystic ovary syndrome, endometriosis stage III/IV or partners with severe male factor requiring ICSI were not included in the study. Likewise, poor responders (previous cycles with $>20$ days of gonadotropin stimulation, or cancellation due to limited follicular response, or $<4$ follicles of $15 \mathrm{~mm}$ ) and patients with a previous IVF cycle with unsuccessful fertilization were excluded from participation.

The primary end point was the clinical pregnancy rate per patient and secondary outcome end points were the number of cumulus-oocyte complexes (COCs) retrieved, the number of metaphase II oocytes obtained, fertilization rate and serum E2 levels and endometrial thickness on the day of hCG administration. We also compared pregnancy loss (including biochemical pregnancies, miscarriages and ectopic pregnancies), implantation rate and ovarian hyperstimulation syndrome (OHSS) rate.

Patients underwent controlled ovarian hyperstimulation following downregulation with a GnRH agonist in a long protocol for women undergoing IVF. All patients received identical type and dose of concomitant fertility treatments, i.e. GnRH agonist for downregulation, hCG for triggering final maturation and progesterone for luteal support. Treatment with GnRH-a [daily SC injections of $0.1 \mathrm{mg}$ triptorelin acetate (decapeptyl; Ferring Pharmaceuticals $\mathrm{GmbH}$ )] was started in the midluteal phase of the menstrual cycle and continued until the day of hCG injection. Ovarian stimulation was started with highly purified hMG (Humog HP, Bharat Serums) or recombinant FSH (follitropin alfa,
GONAL-F; Serono, Geneva, Switzerland or follitropin beta, Puregon; Organon, Holland) on the third day of menstrual bleeding after pituitary desensitization (serum E2 of $<50$ $\mathrm{pg} / \mathrm{ml}$ ) in the absence of an ovarian cyst (diameter of $>2$ $\mathrm{cm}$ ). If such a cyst appeared persistent during the treatment of the GnRH-a and E2 levels did not drop below $50 \mathrm{pg} / \mathrm{ml}$ within 2 weeks after the menstrual bleeding, the patient were excluded from the study.

The starting dose of HP-hMG or r-FSH was 225 IU for the first 5 days, followed by individual adjustments according to the patient's follicular response.

Chorion gonadotropin alfa, $250 \mu \mathrm{g}$ sc (Ovitrelle; Serono, Geneva, Switzerland), was administered to induce final follicular maturation within 1 day of observing three or more follicles of $17 \mathrm{~mm}$ diameter. Oocyte retrieval took place 36 \pm 1 hour after hCG administration. Oocytes were cultured individually (1 oocyte per well or per droplet) throughout culture, from the time of retrieval until the assessment on day 3, allowing for continued individual assessment of each oocyte/embryo. Transfer of one to three embryos fulfilling at least the minimum quality criteria was done on day 2 or 3 after oocyte retrieval. Vaginal progesterone gel $90 \mathrm{mg} /$ day $8 \%$ (Crinone; Serono) for luteal support was given from the day of embryo transfer till confirmation of clinical pregnancy (5-6 weeks after embryo transfer) or negative serum hCG test (13-15 days after embryo transfer). Implantation rate is defined as the total number of gestational sacs in the study divided by the total number of embryos transferred in the study. Clinical pregnancy rate was defined as the presence of a gestational sac with a positive heartbeat 4 to 5 weeks after embryo transfer.

\section{STATISTICAL EVALUATION}

Data were expressed as mean \pm SD. Normality of distribution of continuous variables was assessed with a Kolmogorov-Smirnov test. Between group differences of normally distributed, continuous variables were assessed with parametric statistics (Student's t-test), while nonparametric statistics (Mann-Whitney rank sum test) were employed when the normality test was not passed. In between group differences in noncontinuous variables were assessed with the $\chi^{2}$ method with Yates' correction, if needed.

\section{RESULTS}

Demographic and baseline hormonal profile characteristics of the study participants were similar in both groups (Table 1).

Out of 240 patients initially recruited for the study, eight did not reach the oocyte retrieval procedure $[5$ patients receiving $\mathrm{rFSH}(4.2 \%)$ and 3 having HP-hMG (2.5\%); 
$(\mathrm{p}=0.87)]$. Four patients $(4.1 \%)$ were cancelled because of the low response and four patients (3.8\%) were cancelled for being at risk of OHSS in both groups. Cycle cancellation rates were not significantly different.

Ovarian stimulation outcome are presented in Table 2. Treatment durations and gonadotropin doses were similar in both groups.

In Table 3 parameters of oocyte retrieval and of retrieved oocytes are presented. Total and metaphase II oocytes retrieved, fertilization rate, total number of grade 1 embryos on day 3 , number of embryos transferred were all similar in both groups. The rate of mature oocytes relative to the total number of oocytes retrieved, the embryo cleavage rate and the rate of grade 1 embryos relative to the number of fertilized oocytes were also similar. Out of the 115 patients receiving r-FSH in two patients (1.7\%) and out of the 117 patients receiving HP-hMG in 3 patients $(2.6 \%)$ fertilization failure occurred $(p=0.87)$. Embryo transfer were cancelled in four patients (3.5\%) receiving $\mathrm{r}-\mathrm{FSH}$ and in five patients (4.4\%) receiving HP-hMG because of the low embryo quality in both groups. Cycle cancellation rates were not significantly different.
Implantation rate, clinical pregnancy, pregnancy loss and live birth rates are presented in Table 4. No significant differences were observed between the groups.

\section{DISCUSSION}

At present, different gonadotropin preparations are used in pituitary-suppressed women who are undergoing controlled ovarian stimulation for IVF procedures. Several randomized, prospective trials, comparing the effect of FSHonly and $h M G$ preparations in IVF by using a long GnRH- $\alpha$ protocol, have shown that severe suppression of serum LH levels (1 IU/1) may occur in about half of the FSH-treated subjects. ${ }^{19}$ Although follicular growth can be induced by FSH in the total absence of $\mathrm{LH}$, the resulting follicles have developmental deficiencies such as abnormally low production of estradiol (E2) and an inability to luteinize and rupture in response to an hCG stimulus. ${ }^{20-23}$ Optimal follicular development is, therefore, also dependent on a minimal exposure to LH or 'LH threshold'.

In the Cochrane meta-analyses on the effectiveness of hMG and r-FSH in IVF ICSI cycles, it became evident that hMG treatment resulted in a higher clinical pregnancy rate

\begin{tabular}{|lccc|}
\hline \multicolumn{4}{|c|}{ Table 1: Demographic characteristics and baseline hormonal profiles of the participants } \\
\hline Baseline parameters & $r$-FSH $(n=120)$ & $H P$ - $h M G(n=120)$ & $p$-value \\
\hline Age (years) & $28.1 \pm 3.3$ & $28.2 \pm 2.8$ & 0.91 \\
Body mass index $\left(\mathrm{m} / \mathrm{Kg}^{2}\right)$ & $24.2 \pm 1.5$ & $23.9 \pm 1.7$ & 0.87 \\
Basal FSH (IU/l) & $6.2 \pm 1.8$ & $6.8+1.6$ & 0.74 \\
Basal E2 $(\mathrm{pg} / \mathrm{ml})$ & $39.4 \pm 23.3$ & $39.5 \pm 22.3$ & 0.77 \\
TSH (mIU/l) & $1.8 \pm 0.8$ & $1.9 \pm 1.1$ & 0.86 \\
Antral follicle count $(2-10 \mathrm{~mm})$ & $15.9 \pm 3.9$ & $15.2 \pm 4.1$ & 0.67 \\
\hline
\end{tabular}

Values are expressed by mean \pm SD; r-FSH: Recombinant FSH; HP-hMG: Highly purified hMG; E2: Estradiol

\begin{tabular}{|c|c|c|c|}
\hline & $r-F S H(n=120)$ & $H P-h M G(n=120)$ & $p$-value \\
\hline Total Gn dose (IU) & $2096 \pm 923$ & $2481 \pm 994$ & 0.14 \\
\hline Duration of stimulation (days) & $8.4 \pm 1.6$ & $8.8 \pm 1.5$ & 0.31 \\
\hline Peak estradiol $(\mathrm{pg} / \mathrm{ml})$ & $2292 \pm 965$ & $2444 \pm 978$ & 0.12 \\
\hline Endometrial thickness (mm) & $11.2 \pm 4.3$ & $10.7 \pm \quad 4$ & 0.23 \\
\hline
\end{tabular}

Values are expressed by mean $\pm \mathrm{SD}$

Table 3: Parameters of oocyte retrieval and of retrieved oocytes

\begin{tabular}{lrrr}
\multicolumn{4}{c}{ Table 3: Parameters of oocyte retrieval and of retrieved oocytes } \\
\hline & $r$-FSH $(n=115)$ & HP-hMG $(n=117)$ & $p$-value \\
\hline Total number of oocytes collected & $11.4 \pm 8.1$ & $10.3 \pm 6.0$ & 0.71 \\
Number of metaphase II & $8.7 \pm 6.0$ & $7.8 \pm 4.0$ & 0.43 \\
Metaphase II/total number of oocytes (\%) & $75.5 \pm 20.8$ & $70.1 \pm 18.4$ & $5.1 \pm 3.7$ \\
Number of fertilized oocytes & $6.1 \pm 3.4$ & $72.8 \pm 26.4$ & 0.21 \\
Fertilization rate (\%) & $68.9 \pm 22.3$ & $3.4 \pm 2.7$ & 0.35 \\
Number of grade 1 embryos & $3.5 \pm 2.5$ & $59 \pm 22$ & 2.32 \\
Grade 1 embryos/number of fertilized oocytes (\%) & $52.6 \pm 26$ & $2.3 \pm 0.6$ & 0.26 \\
Number of transferred embryos & $2.6 \pm 0.7$ & 0.08 \\
\hline
\end{tabular}

Values are expressed by mean $\pm \mathrm{SD}$; Fertilization rates are expressed as the mean of (number of zygotes per cycle number of oocytes per cycle) \pm standard deviation for all cycles in which oocytes were retrieved 


\begin{tabular}{lccc}
\multicolumn{4}{c}{ Table 4: Results of cycle outcomes/embryo transfer } \\
\hline & $r$-FSH $(n=109)$ & $H P$-hMG $(n=109)$ & $p$-value \\
\hline Implantation rate (\%) & 36.2 & 38 & 0.88 \\
Positive hCG (\%) & $52.3(57 / 109)$ & $53.2(58 / 109)$ & 0.99 \\
Clinical pregnancy (\%) & $40.4(44 / 109)$ & $45.9(50 / 109)$ & 0.49 \\
Pregnancy loss (\%) & $16(7 / 44)$ & $16(8 / 50)$ & 0.79 \\
Ectopic pregnancy (\%) & - & $2(1 / 50)$ & 0.95 \\
Live birth rate (\%) & $32.1(35 / 109)$ & $37.6(41 / 109)$ & 0.48 \\
\hline
\end{tabular}

and in higher ongoing pregnancy and live birth rates than did r-FSH but the latter difference was of borderline significance. ${ }^{16}$ However, the heterogeneous pituitary suppression regimens and the flexible gonadotropin dosages used in these studies limited the potential for discriminating the features of these two gonadotropin preparations. The importance of using a similar gonadotropin dose was confirmed by the authors of the Cochrane review of 2003. The present clinical study represents a comprehensive and systematic evaluation of oocyte and embryo quality and pregnancy outcome in patients undergoing ovarian stimulation with two different gonadotropin preparations, following a similar stimulation protocol and a similar starting gonadotropin dose.

In the study of Hompes et $\mathrm{al}^{13}$ at an equal dose, HP-hMG displayed a milder stimulation pattern, reflected in a higher cancellation rate as a result of poor ovarian response. Despite the lower number of oocytes retrieved, HP-hMG treatment resulted in a similar ongoing pregnancy rate per started cycle and in a slightly higher ongoing pregnancy rate per transfer (nor reaching statistical significance), as compared with r-FSH. In two large studies performed that compared HP-hMG and r-FSH in a long down regulation protocol for ICSI, the OHSS incidences were similar in both treatment groups. ${ }^{10,11}$ In these studies, however, the dosage could be individually adjusted after 5 days of treatment. Another recent study ${ }^{24}$ that compared HP-hMG and r-FSH for ovulation induction demonstrated that the LH activity in HP-hMG induces a more modulated folliculogenesis that is associated with a lower risk of excessive ovarian response and an ovulation rate similar to that obtained with r-FSH. In the present study no statistically significant or clinically relevant differences were found between the two treatment groups for any of the clinical end points. Comparable follicular development and E2 levels were obtained during stimulation with HP-hMG and r-FSH. Cancellation rates of both groups because of the low response or for being at risk of OHSS were similar.

The results of the current study do not demonstrate significant differences with respect to oocyte, embryo quality and clinical parameters with HP-hMG vs r-FSH in patients whose indication for assisted reproduction was the moderate male factor. The rate of mature oocytes relative to the total number of oocytes retrieved, the embryo cleavage rate and the rate of grade 1 embryos relative to the number of fertilized oocytes were also similar. However, little is known about the quality of the oocytes retrieved and their developmental potential. Limited data from randomized controlled trials are available in the clinical area regarding the impact of LH activity on embryo quality; however, a recent study ${ }^{25}$ reported a higher incidence of grade 1 and 2 embryos when supplementing LH activity to FSH stimulation in women undergoing a long agonist protocol. The mechanisms for the improved oocyte/embryo quality in IVF cycles after exposure to exogenous LH activity are not fully understood, but it has been hypothesized that it could materialize through cumulus cells when exposed to LH activity during stimulation. ${ }^{26}$ Recent gene expression data supported this concept and provided some molecular evidence for a mediation of the cumulus cells in embryo development. ${ }^{27}$

The clinical pregnancy and live birth rates were slightly higher in HP-hMG treated patients but the differences did not reach as statistically significant level. The results of our trial are very similar to the results of the Cochrane metaanalysis ${ }^{16}$ and recent trials comparing HP-hMG with r-FSH in IVF. ${ }^{10,11}$ A higher ongoing pregnancy rate with HP-hMG, compared with FSH, was found but did not reach statistical significance. Another recent study ${ }^{9}$ did use fixed gonadotropin dosage $(150 \mathrm{IU} / \mathrm{d})$. However, because small number of patients were used (50 patients each group), they found no statistically significant difference in reproductive outcomes. It is important to perform more studies to confirm the same results when comparing HP-hMG with r-FSH.

Westerggard et $\mathrm{al}^{28}$ compared the effectiveness of hMG with $\mathrm{rFSH}$ in ovarian stimulation protocols in IVF or ICSI treatment of infertility in normogonadotrophic women in a recent meta-analysis. There was no evidence of a difference between $\mathrm{hMG}$ and r-FSH in ongoing pregnancy/ live birth per woman (OR, 1.27; 95\% CI, 0.98-1.64). Furthermore there was no clear difference on any of the secondary outcomes, although the clinical pregnancy rate per woman was of borderline significance in favor of $\mathrm{hMG}$ (summary OR, 1.28; 95\% CI, 1.00-1.64). The authors 
concluded that more large randomized trials are needed to estimate the difference between hMG and $\mathrm{rFSH}$ more precisely. Such trials should preferably: (1) use a consistent long GnRHa protocol, (2) use a fixed dose of gonadotropin to prevent potentially subjective decisions of the clinician in dosing and (3) take live birth as primary end point. They suggested at this moment in time, however, in prescribing gonadotropins for ovarian hyperstimulation in IVF one should use the least expensive medication. The present study used a consistent long $\mathrm{GnRH}-\alpha$ protocol, used a fixed dose of gonadotropin and take live birth rates as primary end point. The only limitation of the present study might be the small number of patients. The significance of the different pharmacodynamic profiles of these two gonadotropins to the reproductive outcome should be further investigated by even efficacy trials or by other meta-analyses.

\section{CONCLUSION}

We compared the efficacy of HP-hMG vs r-FSH treatments following $\mathrm{GnRH}$ agonist suppression in patients undergoing ICSI with moderate male factor infertility in a prospective randomized controlled trial. We found HP-hMG to be as effective as r-FSH in terms of oocyte and embryo quality and clinical pregnancy outcomes.

\section{REFERENCES}

1. Siristatidis C, Trivella M, Chrelias C, Sioulas VD, Vrachnis N, Kassanos D. A short narrative review of the feasibility of adopting mild ovarian stimulation for IVF as the current standard of care. Arch Gynecol Obstet 2012 Aug;286(2):505-10.

2. Pouwer AW, Farquhar C, Kremer JA. Long-acting FSH versus daily FSH for women undergoing assisted reproduction. Cochrane Database Syst Rev 2012;13;6:CD009577.

3. Reindollar RH, Goldman MB. Gonadotropin therapy: A 20th century relic. Fertil Steril 2012;97:813-18.

4. Beall SA, DeCherney A. History and challenges surrounding ovarian stimulation in the treatment of infertility. Fertil Steril 2012;97:795-801.

5. Loumaye E, Engrand P, Shoham Z, Hillier SG, Baird DT. Clinical evidence of an $\mathrm{LH}$ ceiling effect induced by administration of recombinant human LH during the late follicular phase of stimulation cycles in World Health Organization type I and type II anovulation. Hum Reprod 2003;18: 314-22.

6. Bjercke S, Tanbo T, Abyholm T, Omland A, Opøien HK, Fedorcsak P. Clinical outcome following stimulation with highly purified hMG or recombinant FSH in patients undergoing their first treatment cycle of IVF or ICSI. Acta Obstet Gynecol Scand 2010;89:1053-60.

7. Pacchiarotti A, Sbracia M, Frega A, Selman H, Rinaldi L, Pacchiarotti A. Urinary hMG (Meropur) versus recombinant FSH plus recombinant LH (Pergoveris) in IVF: A multicenter, prospective, randomized controlled trial. Fertil Steril 2010;94: 2467-69.
8. Ye H, Huang G, Pei L, Zeng P, Luo X. Outcome of in vitro fertilization following stimulation with highly purified $\mathrm{hMG}$ or recombinant FSH in downregulated women of advanced reproductive age: A prospective, randomized and controlled trial. Gynecol Endocrinol 2012;28:540-44

9. Kilani Z, Dakkak A, Ghunaim S, Cognigni Ge, Tabarelli C, Parmegiani L, et al. A prospective, randomized, controlled trial comparing HP-hMG with r-FSH in women undergoing ICSI: Ovarian response and clinical outcomes. Hum Reprod 2003;18: 1194-99.

10. European and Israeli study group on highly purified metropin versus r-FSH. Efficacy and safety of highly purified metropin versus r-FSH in IVF, ICSI cycles: A randomized, comparative trial. Fertil Steril 2002;78:520-28.

11. Anderson AN, Devroey P, Arce JC. Clinical outcome following stimulation with HP-hMG or r-FSH in patient undergoing IVF: A randomized assessor blind controlled trial. Hum Reprod 2006;21:3217-27.

12. Ziebe S, Lundin K, Janssens R, Helmgaard L. Joan-Carles Arce for the MERIT (Menotrophin vs Recombinant FSH in vitro Fertilisation Trial) Group. Influence of ovarian stimulation with HP-hMG or recombinant FSH on embryo quality parameters in patients undergoing IVF. Hum Reprod 2007;22:2404-13.

13. Hompes PG, Broekmans FJ, Hoozemans DA, Schats R. For the firm group. Effectiveness of highly purified human menopausal gonadotropin vs recombinant follicle-stimulating hormone in first-cycle in vitro fertilization-intracytoplasmic sperm injection patients. Fertil Steril 2008;89:1685-93.

14. Al-Inany H, Aboulghar M, Mansour R, Serour G. Meta-analysis of recombinant versus urinary-derived FSH: An update. Hum Reprod 2003;18:305-13.

15. Al-Inany H, Aboulghar MA, Mansour RT, Serour GI. Ovulation induction in the new millennium: Recombinant folliclestimulating hormone versus human menopausal gonadotropin. Gynecol Endocrinol 2005;20:161-69.

16. Van Wely M, Westergaard LG, Bossuyt PM, Van der Veen F. Human menopausal gonadotropin versus recombinant follicle stimulation hormone for ovarian stimulation in assisted reproductive cycles. Cochrane Database Syst Rev 2003;(1): CD003973.

17. Al-Inany HG, Abou-Setta AM, Aboulghar MA, Mansour RT, Serour GI. Efficacy and safety of human menopausal gonadotrophins versus recombinant FSH: A meta-analysis. RBM Online 2008;16:81-88

18. World Health Organization. WHO laboratory manual for the examination of human semen and semen-cervical mucus interaction, (5th ed). WHO Press, World Health Organization, 2010.

19. Fleming R, Chung CC, Yates RW, Coutts JR. Purified urinary follicle stimulating hormone induces different hormone profiles compared with menotropins, dependent upon the route of administration and endogenous luteinizing hormone activity. Hum Reprod 1996;11:1854-58.

20. Couzinet B, Lestrat N, Brailly S, Forest M, Schaison G. Stimulation of ovarian follicular maturation with pure follicle stimulating hormone in women with gonadotropin deficiency. J Clin Endocrinol Metab 1988;66:552-56.

21. Shoham Z, Balen A, Patel A, Jacobs HS. Results of ovarian induction using human menopausal gonadotropin or purified follicle-stimulating hormone in hypogonadotropic hypogonadism patients. Fertil Steril 1991;56:1048-53. 
22. Shoham Z, Mannaerts B, Insler V, Coelingh-Bennink H. Induction of follicular growth using recombinant human folliclestimulating hormone in two volunteer women with hypogonadotropic hypogonadism. Fertil Steril 1993;59:738-42.

23. Recombinant human luteinizing hormone (LH) to support recombinant human follicle stimulating hormone (FSH)-induced follicular development in LH- and FSH-deficient anovulatory women: A dose-finding study. J Clin Endocrinol Metab 1998;83: 1507-14.

24. Platteau P, Andersen AN, Balen A, DEvroey P, Sorenson P, Helmgaard L, et al. Similar ovulation rates, but different follicular development with highly purified metropin compared with r-FSH in WHO group 11 anovulatory infertility: A randomized controlled study. Hum Reprod 2006;21:1798-804.

25. Lisi F, Rinaldi L, Fishel S, Caserta D, Lisi R, Campbell A. Evaluation of two doses of recombinant luteinizing hormone supplementation in an unselected group of women undergoing follicular stimulation for in vitro fertilization. Fertil Steril 2005; 83:309-15.

26. Platteau P, Smitz J, Albano C, Sørensen P, Arce J-C, Devroey P. Exogenous luteinizing hormone activity may influence the treatment outcome in in vitro fertilization but not in intracytoplasmic sperm injection cycles. Fertil Steril 2004;81:1401-04.

27. Assou S, Anahory T, Pantesco V, Le Carrour T, Pellestor F, Klein B, et al. The human cumulus-oocyte complex geneexpression profile. Hum Reprod 2006;21:1705-19.
28. Westergaard LW, Bossuyt PM, Van der Veen F, van Wely M. Withdrawn: Human menopausal gonadotropin versus recombinant follicle stimulation hormone for ovarian stimulation in assisted reproductive cycles. Cochrane Database Syst Rev 2011;16(2):CD003973.

\section{ABOUT THE AUTHORS}

\section{Rutvij Jay Dalal}

Super Specialty Fellow in Reproductive Medicine, Department of IVF Lilavati Hospital and Research Centre, Mumbai, Maharashtra, India

Corresponding Address: 20, Shreerang Society, Near Old Pilot Dairy, Kankaria, Ahmedabad-380022, Gujarat, India Phone: +91-9611057700, e-mail: rutvij.dalal@gmail.com

\section{Hrishikesh Pai}

Senior Consultant, Department of IVF, Lilavati Hospital and Research Centre, Mumbai, Maharashtra, India

\section{Nandita Palshetkar}

Senior Consultant, Department of IVF, Lilavati Hospital and Research Centre, Mumbai, Maharashtra, India 